\title{
PAH AND DUST PROCESSING IN SUPERNOVA REMNANTS
}

\author{
J. Rho ${ }^{1}$, M. Andersen ${ }^{2}$, A. Tappe ${ }^{3}$, W.T. Reach ${ }^{1}$, J.P. Bernard ${ }^{4}$ \\ and J. Hewitt ${ }^{5}$
}

\begin{abstract}
I present observations of shock-processed PAHs and dust in supernova remnants (SNRs). Supernova shocks are one of the primary sites destroying, fragmenting and altering interstellar PAHs and dust. Studies of PAHs through supernova shocks had been limited because of confusion with PAHs in background emission. Spitzer observations with high sensitivity and resolution allow us to separate PAHs associated with the SNRs and unrelated, Galactic PAHs. In the young SNR N132D, PAH features are detected with a higher PAH ratio of 15-20/7.7 $\mu \mathrm{m}$ than those of other astronomical objects, and we suggest large PAHs have survived behind the shock. We present the spectra of additional 14 SNRs observed with Spitzer IRS and MIPS SED covering the range of $5-90 \mu \mathrm{m}$. Bright PAH features from 6.2 to $15-20 \mu \mathrm{m}$ are detected from many of SNRs which emit molecular hydrogen lines, indicating that both large and small PAHs survive in low velocity shocks. We observe a strong correlation between PAH detection and carbonaceous small grains, while a few SNRs with dominant silicate dust lack $\mathrm{PAH}$ features. We characterize PAHs depending on the shock velocity, preshock density and temperature of hot gas, and discuss PAH and dust processing in shocks and implication of $\mathrm{PAH}$ and dust cycles in ISM.
\end{abstract}

1 SOFIA Science Mission Operations/USRA, NASA Ames Research Center, MS 211-3, Moffett Field, CA 94035, USA; e-mail: jrho@sofia.usra.edu

2 Research \& Scientific Support Department, European Space Agency, ESTEC, Keplerlaan 1, 2200 AG Noordwijk, The Netherlands

3 Harvard-Smithsonian Center for Astrophysics, MS 83, 60 Garden Street, Cambridge, MA 02138, USA

${ }^{4}$ Centre d'Étude Spatiale des Rayonnements, CNRS, 9 Av. du Colonel Roche, BP. 4346, 31028 Toulouse, France

5 NASA/Goddard Space Flight Center, Code 662, Greenbelt, MD 20771, USA 


\section{Introduction}

Supernova shocks are one of the primary sites destroying, fragmenting and altering interstellar polycyclic aromatic hydrocarbons (PAHs) and dust. Supernovae influence the chemistry and physics in the interstellar medium (ISM) from galactic scales down to the atomic level. Studies of PAHs through supernova shocks had been limited because of confusion with PAHs in background emission. Spitzer observations with high sensitivity and resolution allow us to separate PAHs associated with the supernova remnants (SNRs) and unrelated, Galactic PAHs. Moreover, ISO observations of the continuum were limited to wavelengths longer than $40 \mu \mathrm{m}$ and the dust analysis was focused on the big grains. Shorter wavelength observations are necessary to probe the emission from the very small grains and polycyclic aromatic hydrocarbons (PAHs) in greater details. The ratio of $\mathrm{PAH}$ and very small grain (VSG) to big grain (BG) abundance can provide insight of the dust destruction mechanisms.

Dust grains and PAHs control the heating and cooling balance of interstellar gas through absorption of ultraviolet radiation, generation of photoelectrons, and emission of infrared radiation. These processes allow us to use them as diagnostics to probe physical conditions in a range of diverse environments. However, this is hampered by our lack of knowledge of their exact structure and composition, and how these are in turn affected by the physical environment. The PAHs are believed to give rise to the unidentified infrared bands (UIR), well-known emission features near 3.3, 6.2, 7.7, 8.6, and $11.2 \mu \mathrm{m}$ attributed to the CC and CH stretching and bending modes of these molecules (Allamandola et al. 1989). More recently observed features at 15-20 $\mu \mathrm{m}$ are interpreted as PAH CCC out-of-plane bending modes (Peeters et al. 2004). Several detections have been reported in a variety of astronomical objects.

We present observations of shock-processed PAHs and dust in SNRs with Spitzer IRAC and MIPS imaging and IRS and MIPS-SED spectroscopy covering from $3.6 \mu \mathrm{m}$ to $95 \mu \mathrm{m}$. We confirm the PAH detection and its distribution in the young SNR N132D and present GLIMPSE-selected (Reach et al. 2006) 14 SNRs, many of which are interacting molecular clouds. We characterize PAHs depending on a shock velocity, preshock density and temperature of hot gas, and discuss correlation of PAH and associated dust composition and dust cycles in ISM.

\section{PAH detection from the supernova remnant N132D}

N132D is a young SNR in the Large Magellanic Cloud (LMC). The age of N132D is $2500 \mathrm{yr}$. In the optical, the large-scale morphology of the gas in N132D shows highly red-shifted oxygen-rich ejecta in the center and shocked interstellar clouds in the outer rim. We have previously presented Spitzer IRAC, MIPS and an IRS spectrum of N132D (Tappe et al. 2006). Here we present IRS-LL mapping of N132D covering the entire SNR, and IRS staring of SL observations toward 4 positions. 


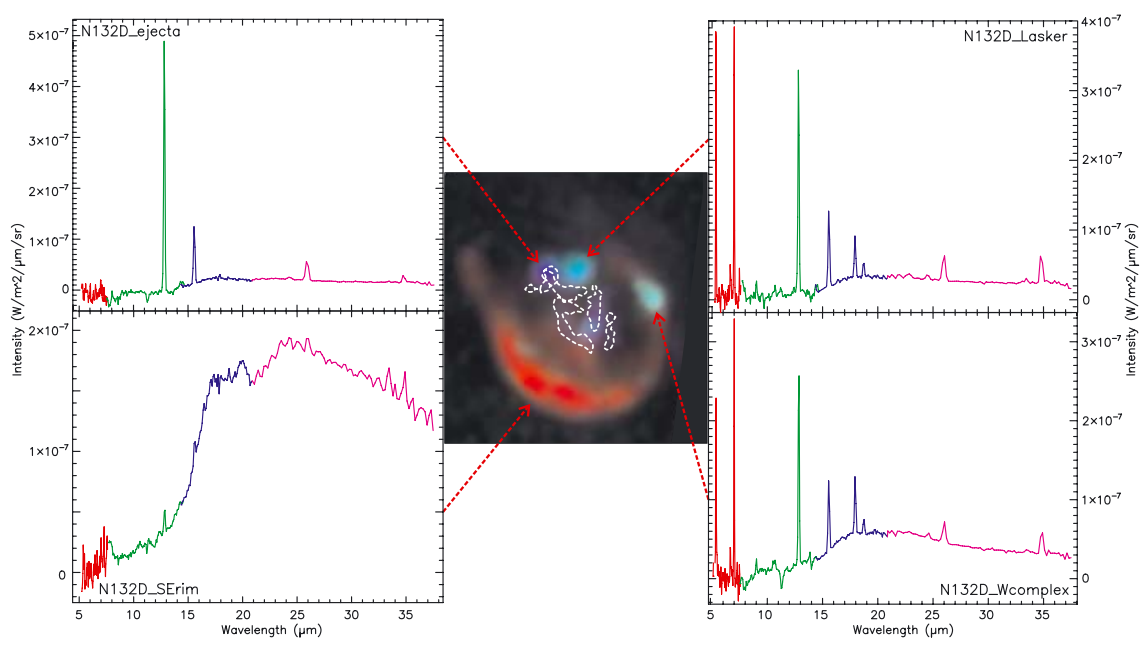

Fig. 1. N132D spectral mapping (center; blue $=[$ Ne III $] 15.5 \mu \mathrm{m}$, green $=[$ Fe II $] 17.9 \mu \mathrm{m}$, red $=$ MIPS $24 \mu \mathrm{m}$, white dashed line $=$ red and blueshifted high-velocity $\mathrm{Ne} / \mathrm{O}$ ejecta seen in the optical with HST) and spectra of selected positions.

We detect bright 24 and $70 \mu \mathrm{m}$ emission from shocked, heated dust grains with MIPS. The 5-40 $\mu \mathrm{m}$ IRS mapping was performed, where LL $(13-40 \mu \mathrm{m})$ covered the entire SNR and SL $(5-13 \mu \mathrm{m})$ covered a few interesting positions. Figure 1 shows representative sets of spectra from southern rim (bottom left spectrum), the West Complex (bottom right), Lasker Bowl (top right) and an ejecta knot (top left).

IRS spectrum toward the southeastern shell shows a steeply rising continuum with [Ne III] and [O IV] as well as PAH features at $\sim 7.7,11.3$, and $15-20 \mu \mathrm{m}$. The PAH bands are from swept-up ISM material, which are processed by the strong shock wave via thermal sputtering and grain-grain collisions (Tielens et al. 1994). We found evidence for spatial variations of the PAH features, both in intensity and wavelength, when comparing spectra extracted along the IRS long-low slit from the shell of the remnant and the adjacent molecular cloud/H II region, which confirms the processing of the PAH molecules. These detections, most notably the $15 \sim 20 \mu \mathrm{m}$ features, are the first of its kind for supernova remnants to our knowledge (Tappe et al. 2006). The $15 \sim 20 \mu \mathrm{m}$ features in Figure $2 \mathrm{a}$ are interpreted as PAH CCC out-of-plane bending modes ( $c f$. van Kerckhoven et al. 2000; Peeters et al. 2004). We generated a PAH map between 15-20 $\mu \mathrm{m}$ (Fig. 2b) after subtracting the continuum at neighboring wavelengths; the morphology is similar to that of the dust continuum in the MIPS $24 \mu \mathrm{m}$ band (Fig. 1). Several detections of $15-20 \mu \mathrm{m}$ PAH features have been reported in a variety of sources, including H II regions, young stellar objects, reflection nebulae, and evolved stars (see Peeters et al. 2004, for references therein). The detections include both broad variable plateaus as well as distinct, narrow features, most notably at 16.4 and $17.4 \mu \mathrm{m}$. 

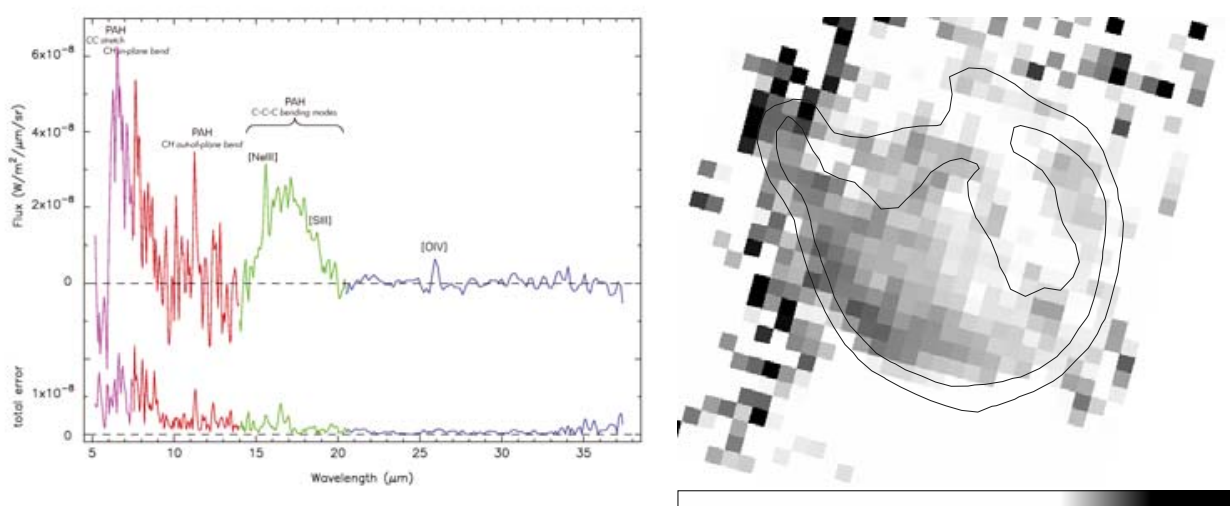

Fig. 2. (a: Left) N132D southeastern rim spectrum (magenta line = SL2, red line = SL1, green line $=$ LL2, blue line = LL1): the local background and the modified blackbody continuum have been subtracted. The total error includes the reduction pipeline delivered errors and an estimate of the variation in the local background (Tappe et al. 2006). (b: Right) PAH grey-scale map of N132D between 15-20 $\mu \mathrm{m}$ after subtracting neighboring wavelength dust continuum, superposed on MIPS $24 \mu \mathrm{m}$ contours from Tappe et al. (2006).

\section{PAH from slow shocks: Molecular supernova remnants}

Interacting SNRs are an ideal laboratory to study the effects of fast shocks on the interstellar material. Shocks can both sputter and shatter interstellar dust and thus potentially modify its abundance and size distribution. Previous observations of interacting SNRs had been limited. Using Spitzer GLIMPSE survey data (Reach et al. 2006) identified a series of Galactic SNRs in the infrared, a subsample having IRAC colors that indicate shocked $\mathrm{H}_{2}$. We present follow-up Spitzer IRS low resolution and MIPS SED spectra of a sample of 14 SNRs - G11.2-0.3, G21.8-0.6 (Kes 69), G22.7-0.2, G39.2-0.3 (3C 396), G54.4-0.3, G304.6+0.1 (Kes 17), Kes 20A (G310.8-0.4), G311.5-0.3, G332.4-0.4 (RCW 103), G344.7-0.1, CTB 37A (G348.5+0.1), G348.5-0.0, and G349.7+0.2. Molecular hydrogen detections towards 12 SNRs are presented by Hewitt et al. (2009) and Andersen et al. (2010), more than doubling the sample of known interacting Galactic SNRs. Here we present an analysis of the continuum and PAH emission. The spectroscopy observations were centered on the emission peaks for each SNR. The wavelength coverage from 5 to $90 \mu \mathrm{m}$ ensures a good sampling of the three main dust species and enables us to fit the continuum in greater detail.

The dust model fitting of the SEDs (Fig. 3a) is done using the method described in Bernard et al. (2008). Three dust components are adopted, PAH molecules, Very Small Grains (VSG) and Big Grains (BG). The abundance of each dust species $\left(\mathrm{Y}_{P A H}, \mathrm{Y}_{V S G}, \mathrm{Y}_{B G}\right)$ and the strength of the radiation field $\left(\mathrm{X}_{I S R F}\right)$ are taken 

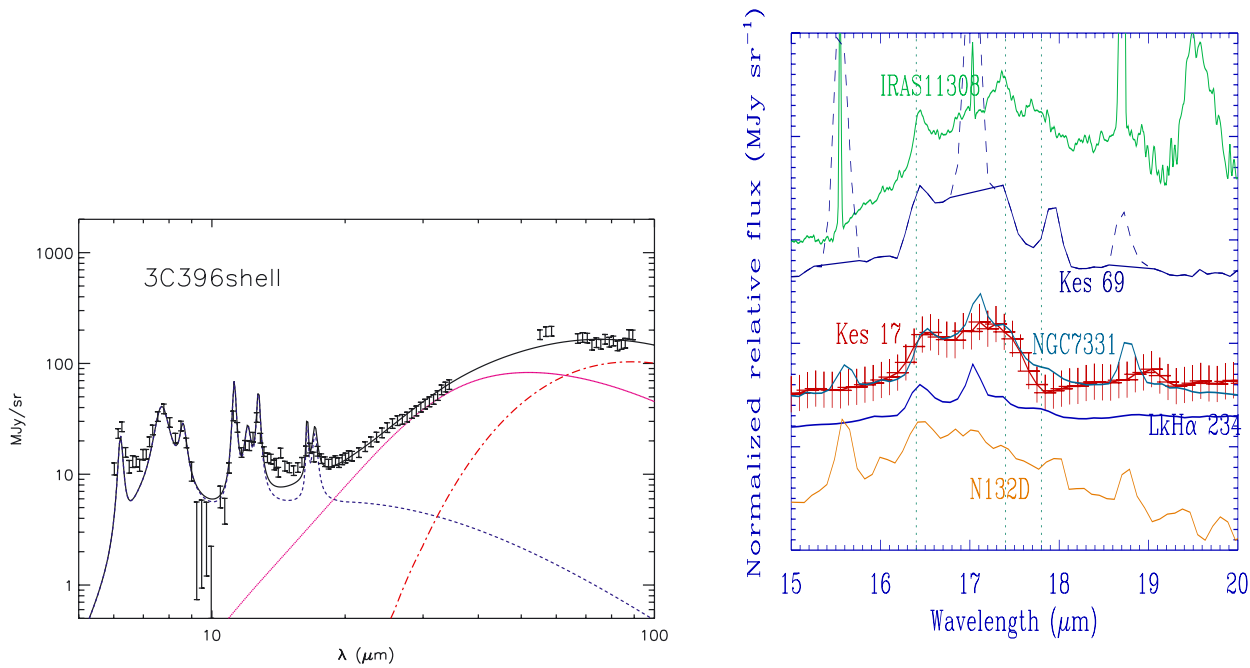

Fig. 3. (a: Left) the DUSTEM fit for 3C 396 superposed IRS and MIPS SED data with 3 component models of PAH, very small grains and big grains. The PAH, very small and large grain contribution are shown as the dotted line, the thin solid line, and the dashed dotted. The total fit is shown as the thick solid line (from Andersen et al. 2010). (b: Right) comparison of PAH emission in the SNR Kes 17 (red curve), Kes 69 (dark blue) and N132D (orange) with those of IRAS 11308 (green), LkH $\alpha 234$ (PDR: blue) and NGC 7331 (yellow).

as free parameters in the fit. For all three dust species, a MRN powerlaw size distribution is assumed. The slopes and size ranges are different than adopted in the original version of DUSTEM due to the updated input physics, mainly the cross sections adopted have changed (Compiégne et al. 2008). The reduced $\chi^{2}$ range between $4-10$. Systematically higher reduced $\chi^{2}$ around the $15-20 \mu \mathrm{m}$ show that the PAH features in SNRs are significantly different from those in PDRs. The fit results are summarized in Figure 4a, showing that abundances of PAHs and VSGs are higher than those in the Milky Way and the LMC (Bernard et al. 2008).

A dust model composed of PAHs, VSGs and BGs provide a good fit to the SEDs of the SNRs. All the SNRs show evidence for PAH emission. Two SNRs, G11.20.3, and G344.7-0.1 show no to little evidence for VSGs and a lower PAH/BG ratio than observed in the diffuse interstellar medium and the LMC. We show Kes 17 and Kes 69 as examples (Fig. 3b) to demonstrate the differences in PAH emission between dust processed by a SNR shock and PDR dominated dust. Typically the radiation field is 10-100 times larger than normal interstellar radiation ISRF, the strength of which is consistent with being created from the shock.

The dust spectral fitting indicates the presence of PAH emission in most of SNRs. In Figure 3b, PAH spectra of Kes 17 and Kes 69 are compared with the 

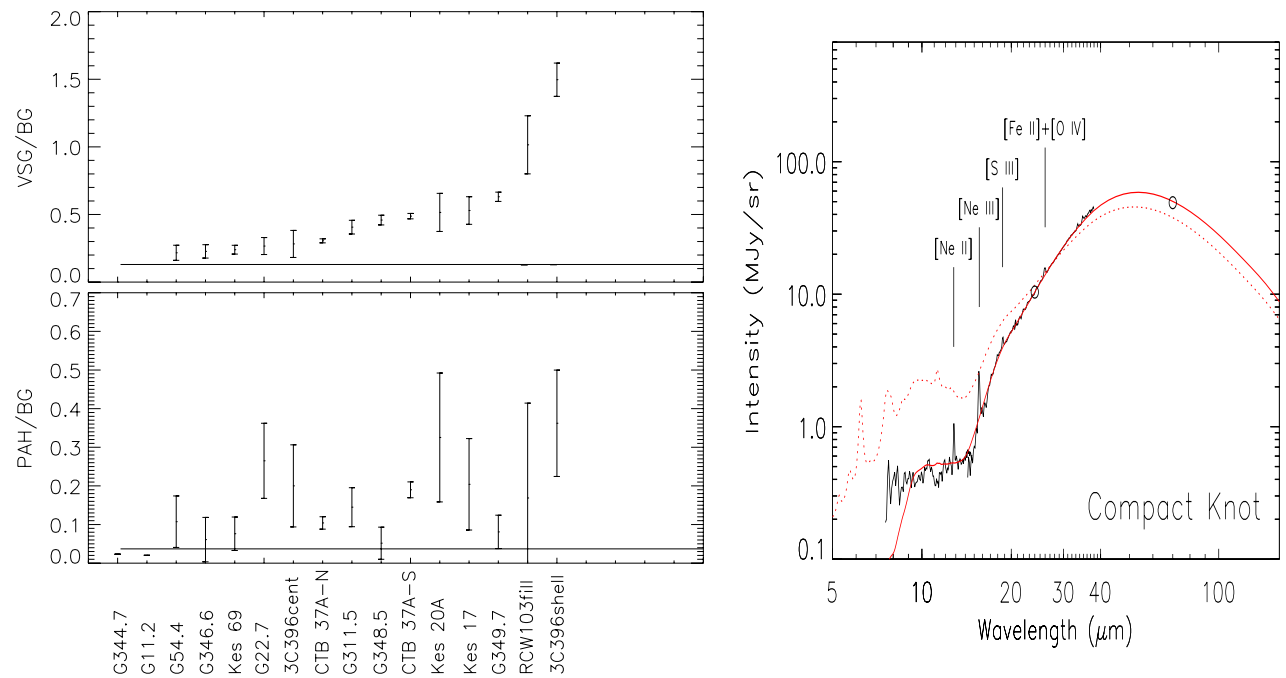

Fig. 4. (a: Left) the ratios of PAHs and very small grains to big grains are higher than those of the Milky Way (solid line). Two young SNRs (G11.2 and G344.2) have the ratios of carbon dust to silicates smaller than the Milky Way value and are associated with a strong radiation field. (b: Right) IRS spectrum of the Puppis A SNR superposed on the best fit (solid line) of the ZDA model (Arendt et al. 2010). The dotted line is a model with PAH and VSG, which shows similarity to the N132D spectrum.

emission from LkH $\alpha 234$ and NGC7331, a PDR and a nearby spiral galaxy with strong PAH emission, respectively. We find that the PAH features of molecular interacting $\left(\mathrm{H}_{2}\right.$ emitting) SNRs are very similar to each other as shown for the cases of Kes 17 and Kes 69. The PAH emission is detected in our background spectra since all the SNRs are located in the Galactic plane. However, the PAH emission is clearly enhanced in the shocked region and is present after background subtraction. The $15-20 \mu \mathrm{m}$ PAH feature in Kes 17 is stronger than that of $\mathrm{LkH} \alpha$ 234 whereas the PAH features between $5-14 \mu \mathrm{m}$ are almost identical. It is similar to that of NGC 7331. The ratio of the emission from the $15-20 \mu \mathrm{m}$ plateau to the $6.2 \mu \mathrm{m}$ PAH feature is $\sim 0.4$ for Kes 17 and other $\mathrm{H}_{2}$ emitting SNRs, similar to NGC 7331 but larger than for the PDR. This ratio is smaller than that of the young SNR N132D (Tappe et al. 2006). The difference in PAH features between molecular SNRs and N132D is due to the environments of SNRs. Many of the interacting SNRs are in a dense molecular cloud environment with a slow shock while N132D is less dense environment and has a strong shock which significantly destroy small PAHs. 


\subsection{Comparison with Puppis $A$ and other dust model}

Figure $3 \mathrm{~b}$ shows IRS spectrum of a middle-age SNR, Puppis A. Spectral fitting of Puppis A (Arendt et al. 2010) was performed based on carbon and silicated grains using Zubko et al. (2004; hereafter ZDA), radiatively heated by an ambient interstellar radiation field. The ZDA models are a set of models for the dust grain size (or mass) distributions that are derived to simultaneously fit average interstellar extinction, emission, and abundances. Different models are distinguished by different choices of grain compositions and different sets of abundance constraints. The PAH emission is not evident in Puppis A. In contrast, PAH features are noticeable in N132D, implying that the grains in N132D is less sputtered than those in Puppis A.

\section{References}

Andersen, M., Rho, J., Tappe, A., et al., 2010, submitted

Arendt, R.G., Dwek, E., Blair, W.P., et al., 2010, ApJ, 725, 585

Bernard, J.P., Reach, W.T., et al., 2008, AJ, 136, 919

Compiégne, M., Abergel, A., Verstraete, L., \& Habart, E., 2008, A\&A, 491, 797

Hewitt, J.W., Rho, J., ndersen, M., \& Reach, W.T., 2009, ApJ, 649, 1266

Peeters, E., Mattioda, A.L., Hudgins, D.M., \& Allamandola, L.J., 2004, ApJ, 617, L65

Reach, W.T., et al., 2006, AJ, 131, 1479

Tappe, A., Rho, J., \& Reach, W.T., 2006, ApJ, 653, 267

van Kerckhoven, C., et al., 2000, A\&A, 357, 1013

Zubko, V., Dwek, E. \& Arendt, R.G., 2004, ApJS, 152, 211 